\title{
LEUKOCYTE AND BACTERIA IMAGING IN PROSTHETIC JOINT INFECTION
}

\author{
Andor W.J.M. Glaudemans ${ }^{1}$, Filippo Galli², Marta Pacilio² and Alberto Signore, ${ }^{1,2, *}$ \\ ${ }^{1}$ Department of Nuclear Medicine and Molecular Imaging, University Medical Centre Groningen, \\ University of Groningen, Groningen, the Netherlands \\ ${ }^{2}$ Nuclear Medicine Unit, Department of Medical-Surgical Sciences and of Translational Medicine, \\ Faculty of Medicine and Psychology, "Sapienza" University, Rome, Italy
}

\begin{abstract}
There has been a significant increase in the number of joint prosthesis replacements worldwide. Although relatively uncommon, complications can occur with the most serious being an infection. Various radiological and nuclear imaging techniques are available to diagnose prosthetic joint infections (PJI). In this review article, we describe the pathophysiology of PJI, the principles of nuclear medicine imaging and the differences between Single Photon Emission Computed Tomography (SPECT) and Positron Emission Tomography (PET). The value of nuclear medicine techniques for clinical practice is also discussed.

Then we provide an overview of the most often used radionuclide imaging techniques that may be helpful in diagnosing prosthetic joint infection: the ${ }^{67} \mathrm{Ga}$-citrate, labelled white blood cells in vitro and in vivo (monoclonal antibodies directed against specific targets on the leukocytes), and ${ }^{18} \mathrm{~F}$-fluorodeoxyglucose $\left({ }^{18} \mathrm{~F}-\mathrm{FDG}\right)$. We describe their working methods, the pitfalls, and the interpretation criteria. Furthermore, we review recent advances in imaging bacteria, a molecular imaging method that holds promises for the detection of occult infections. We conclude proposing two diagnostic flow-charts, based on data in the literature, that could help the clinicians to choose the best nuclear imaging method when they have a patient with suspicion of or with proven PJI.
\end{abstract}

Key words: Prosthetic joint infection; nuclear medicine; fluorodeoxyglucose (FDG); leukocyte imaging; bacteria imaging; white blood cell labelling.

*Address for correspondence:

A. Signore

Medicina Nucleare Unit, Ospedale S. Andrea

University of Rome "Sapienza"

Via di Grottarossa 1035, 00189 Roma, Italy

Telephone Number: +39-06-33775471

E-mail: alberto.signore@uniroma1.it

\section{Introduction}

Over the past years, due to an increased life expectancy, there has been a significant increase in the number of joint prosthesis replacements worldwide. It is a costeffective surgical intervention for osteoarthritis of the hip and knee, results in enhancement of joint function and pain relief (Brunenberg et al., 2005) and is one of the most reliable and cost effective surgical procedures performed (Anguita-Alonso et al., 2005). Although relatively uncommon, complications can occur. The most serious complication of joint prosthesis is infection with an incidence of $1.5-2.5 \%$ for primary interventions and up to $20 \%$ for revision procedures, leading to mortality rates ranging between 1 and nearly $3 \%$ and a significant increase in financial costs (Cataldo et al., 2010).

Prosthetic joint infections (PJI) are usually classified in relation to the time of onset after surgery as "early" (first 3 months after surgery), "delayed" (between 3 months and 2 years after surgery) or "late" (more than 2 years after surgery) (Zimmerli et al., 2004). Host factors predisposing to infection are higher age, poor nutritional status, underlying joint disease (rheumatoid arthritis, psoriasis), diabetes mellitus, obesity, malignancy, remote infection, prior native joint infection, and immune depression (Berbari et al., 1998; Zimmerli et al., 2006; Cataldo et al., 2010). Staphylococcus aureus is usually isolated in early infections whereas coagulase-negative Staphylococci are the main aetiologic agents in late infections as well as Streptococci, Enterococci and Anaerobes (Zimmerli et al., 2004).

Despite the clinical importance of prompt identification of PJI, diagnosis remains difficult. There is still no single diagnostic test available to definitely confirm the presence of PJI. Cultures are the gold standard for diagnosing PJI, with aspiration and/or tissue biopsy used to obtain specimens for additional microbiological investigation. However, the reported sensitivity of these tests varies from 12 to $100 \%$ (Johnson et al., 1988; Duff et al., 1996; Fuerst et al., 2005; Fink et al., 2008; Meermans and Haddad, 2010).

Various imaging techniques are available for the evaluation of the (infected) prosthesis. Radiological techniques, such as plain radiographs, contrast arteriography, and computed tomography (CT) may be helpful, but are not always able to differentiate aseptic from septic prosthetic loosening being based on the presence of indirect signs of infection. Magnetic resonance imaging (MRI) may also recognise infections by using functional probes; however, the metal prostheses can introduce strong artefacts. Several nuclear medicine techniques, by contrast, are able to image leukocytes or bacteria, thus 
providing direct evidence of the inflammatory pattern. They can detect infections of the bone before changes are visible on plain radiographs (Pineda et al., 2009). From data available in the literature and from available metaanalysis, we currently think that nuclear medicine is the imaging modality of choice for the evaluation of suspected PJI.

In this article, we provide an overview of the most often used nuclear medicine imaging techniques that may be helpful in diagnosing PJI. In vitro and in vivo labelled leukocytes, radiopharmaceuticals that are already used for decades $\left({ }^{67} \mathrm{Ga}\right.$-citrate), positron-emitting radiopharmaceuticals ( ${ }^{18} \mathrm{~F}$-Fluorodeoxyglucose) and labelled monoclonal antibodies (developed in recent years) will be explained and discussed. Furthermore, radiopharmaceuticals selectively targeting the causative microorganisms (radiolabelled antibiotics, vitamins and antimicrobial peptides), have recently been developed and will also be reviewed. The methods, pitfalls, and interpretation criteria of the most used radiopharmaceuticals will be explained based on the pathophysiology of PJI.

\section{Pathophysiology of PJI}

Implanted devices always have a higher susceptibility of being colonised by bacteria and fungi, since in animal models it was shown that the presence of a foreign body decreases the minimal infected dose of $S$. aureus leading to a permanent abscess. Most important microorganisms causing implant-associated infections are often skinderived bacteria that can colonise an implant during surgery. Furthermore, since granulocytes around an implant are partially degranulated and have an impaired killing mechanism, they are not able to clear even a small quantity of microorganisms (Zimmerli et al., 1982).

Many microorganisms are able to form biofilms around a device, which is a virulence factor for the development of infections. The formation of a biofilm involves several steps, starting from rapid surface attachment, followed by multi-layered cellular proliferation and intercellular adhesion in a matrix excreted by the bacteria. This initial phase of adherence is followed by an accumulative phase, during which bacterial cells adhere to each other and create a biofilm (Gotz, 2002). Bacteria that lie deep within this biofilm are protected against host phagocytes and are highly resistant to antibiotics. This may explain the indolent nature of prosthetic joint infections and the difficulties that arise in diagnosing, and in eradicating the infection unless the prosthesis is removed (Goldenberg, 1998). Under unfavourable environmental conditions, microorganisms can detach from the biofilm and become free-floating, or planktonic. The presence of planktonic organisms in the bloodstream or urine can lead to sepsis and metastatic infections (Von Eiff et al., 2005).

Biofilms, therefore, allow bacterial growth, reduce leukocyte migration and phagocytosis, and reduce antibiotic penetration and treatment of infections. Bacteria in biofilms are over 1000 times more resistant to antibiotics than are planktonic cells. Radiopharmaceuticals can also poorly penetrate in biofilms, thus sometimes making diagnosis of infection difficult.

From the host's point of view, chemotactic factors secreted by bacteria (mainly in the planktonic growth phase) induce endothelial activation, oedema and recruitment of leukocytes (mainly granulocytes). This continuous recruitment of neutrophils from blood to peri-prosthetic tissue is typical of acute or sub-acute infections in which bacteria grow in both adherent and planktonic growth phases. This is the biological basis for image interpretation when using radiolabelled peripheral blood white blood cells (WBC). Chronic, long-standing, infections (mainly due to chronic presence of bacteria in biofilms) is characterised by a less evident neutrophil recruitment and less oedema. When WBC scintigraphy is performed, delayed imaging after $24 \mathrm{~h}$ is imperative, since the biofilm probably causes a decrease of the invasion of the labelled cells into the infected prosthesis region leading to a more protracted course of WBC accumulation (Larrika et al., 2001). This also explains why a WBC scan is less effective and the use of radiolabelled anti-granulocyte monoclonal antibodies (MoAb) is more indicated, MoAb being facilitated in penetrating into the tissue and binding to infiltrating neutrophils (Richter et al., 2011).

On the other hand, sterile inflammation following a physiological host response to foreign body (prosthesis), or secondary to loosening of the prosthesis, is characterised by a macrophage-lymphocyte infiltration with poor neutrophil migration from blood stream. This explains why, in nuclear medicine, we consider the peri-prosthetic accumulation of radiolabelled granulocytes over time as a surrogate marker of the presence of bacteria - thus infection. A weak accumulation or even a decrease of peri-prosthetic granulocytes over time is considered to be a sign of sterile inflammation.

\section{Clinical relevance of imaging PJI}

Diagnosing a PJI is difficult, most of the time. A delay in the antibiotic and surgical treatment has an important impact on the chance of saving the prosthesis and joint function. It is therefore of invaluable importance to make the diagnosis of PJI as soon as possible.

To date, various nuclear medicine techniques exist to diagnose infections (Chianelli et al., 2008; Signore et al., 2010; Gemmel et al., 2012). The problem is that different countries and different institutes within countries use different radiopharmaceuticals, different imaging techniques and different interpretation criteria. Not all hospitals follow a defined, standard algorithm for when exactly imaging should be performed. Furthermore, there is no consensus between the clinician and the imaging expert concerning at what stage of the diagnostic process nuclear imaging techniques are required. Within the European Association of Nuclear Medicine, a permanent Committee on Imaging of Infection and Inflammation focuses on these problems and consensus guidelines are being produced to reach a worldwide agreement. This paper provides some insights of which possibilities nuclear medicine offers for 
PJI diagnosis. Furthermore, based on data from literature and our experience, we describe diagnostic pathways to decide which technique should be used, at which time point and in which sequence.

\section{Principles of nuclear medicine and the differences between SPECT and PET}

A radiopharmaceutical is a synthetic compound formed by a radioactive isotope and a drug. The isotope, which must not detach from the drug, provides the signal and the biodistribution and targeting characteristics are determined by the drug and must not be altered by the presence of the isotope. For example, the ligand hydroxymethylenediphosphonate (HDP) is preferentially taken up by the bone. As a consequence, by chemically attaching (labelling) radioactive technetium $\left({ }^{99 \mathrm{~m}} \mathrm{Tc}\right)$ to HDP, the radioactive isotope is transported by HDP to the bone for imaging.

A radioisotope is an atom with an unstable nucleus, which undergoes a radioactive decay, emitting rays, which can be imaged using a gamma camera. Different isotopes produce different characteristic photons of a specific energy. For example, ${ }^{99 \mathrm{~m}} \mathrm{Tc}$ emits gamma rays with an energy of $140 \mathrm{keV}$, which can be imaged by a gamma camera. This gamma camera is also able to acquire images from different angles (64 or 128) in a circular manner around a patient and subsequently reconstruct a $3 \mathrm{D}$-image. This is called Single Photon Emission Computed Tomography (SPECT). The resolution of a gamma-camera is around $8 \mathrm{~mm}$.

Other radioisotopes, for example ${ }^{18}$ Fluoride, are positron-emitting isotopes. These positrons will react with an electron in the proximity, thereby undergoing a reaction that is called annihilation, in which the masses of the positron and electron are transformed into energy, forming two gamma photons with the same energy $(511 \mathrm{keV})$ in exactly opposite directions. This process can be depicted by a positron emission tomography (PET) camera that uses a ring detector around a patient and registers only coincidental impacts in opposite parts of the ring detector. The resolution of a PET-camera is nowadays $4-5 \mathrm{~mm}$. Fig. 1 shows the different cameras that are used in nuclear medicine.

Both SPECT and PET new generation cameras are nowadays equipped with CT (SPECT-CT and PET-CT). This provides a unique possibility to image physiology (SPECT and PET) and anatomy (CT) together, thereby leading to better diagnostic accuracy. Furthermore, scan acquisition times are shorter and the patient has only to come to the hospital once (one-stop-shop-principle).

The radiopharmaceuticals that are most often used in imaging infection and inflammation are SPECT radiopharmaceuticals, except for the use of ${ }^{18} \mathrm{~F}$-fluorodeoxyglucose $\left({ }^{18} \mathrm{~F}-\mathrm{FDG}\right)$. The most often used radiopharmaceuticals, their working mechanisms, pros and cons, and interpretation criteria of the derived images are described below.

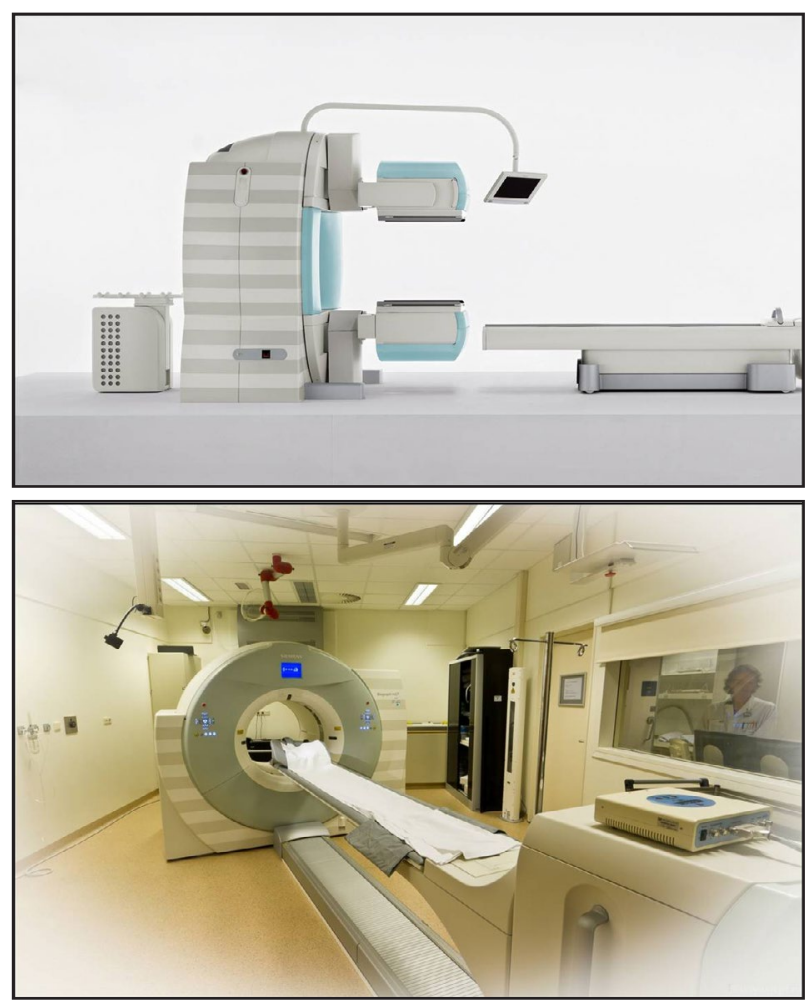

Fig. 1. Upper image: gamma-camera with SPECT/CT (Siemens Symbia T, spatial resolution $8 \mathrm{~mm}$ ), Lower image: PET/CT camera (Siemens Biograph mCT, spatial resolution $4 \mathrm{~mm}$ ).

\section{${ }^{67}$ Ga-citrate, the old gold-standard}

${ }^{67}$ Gallium-citrate $\left({ }^{67} \mathrm{Ga}\right)$ has been used for localising infections for over three decades (Lavender et al., 1971). The exact reason why ${ }^{67} \mathrm{Ga}$ accumulates in infections is still not clarified; however, several factors are governing the accumulation. It binds in ionic form to circulating transferrin as an analogue of iron and uses transferrin receptors (CD71) to access the cell and then becomes highly stable within the cells. Increased blood flow and increased vascular membrane permeability results in increased delivery and accumulation of transferrinbound ${ }^{67} \mathrm{Ga}$ in infected areas. It also binds to lactoferrin, which is present in high concentrations in infected foci. Some ${ }^{67} \mathrm{Ga}$ may even be transported by direct binding to leukocytes, and also direct uptake by certain bacteria has been observed, due to the high affinity of siderophores, low-molecular-weight chelates produced by bacteria, for ${ }^{67} \mathrm{Ga}$. This siderophore- ${ }^{67} \mathrm{Ga}$ complex is, presumably, transported into the bacterium, where it remains until phagocytosed by macrophages (Love and Palestro, 2004). In the first $24 \mathrm{~h}$, approximately $25 \%$ of the total injected dose is excreted by the kidneys. Beyond this time, the large bowel is the principal excretory pathway. The rest is retained in bone, bone marrow, liver and soft tissues (Hoffer, 1980). The best target-to-background ratio is normally achieved after $48-72$ hours. ${ }^{67} \mathrm{Ga}$-citrate is still one the most used radiopharmaceuticals in the world, especially in the United States, for imaging infections. It has however, many disadvantages. Its low specificity, 
long physical half-life and high-energy $\gamma$ radiation are unfavourable characteristics for scintigraphic imaging and cause an increased radiation absorbed dose to patients and personnel (Perkins, 1981; Bekerman et al., 1984). ${ }^{67} \mathrm{Ga}$-citrate has been almost completely replaced by other, more specific radiopharmaceuticals and should not be used anymore in PJI. Therefore, we will not go into details about the methods and interpretation of this imaging technique. Recently, citrate was labelled with the PET radionuclide ${ }^{68} \mathrm{Ga}$, which may, due to the better characteristics of a PET tracer, have potential in the future (Nanni et al., 2010; Kumar et al., 2012).

\section{${ }^{99 m}$ Tc-labelled diphosphonates (bone scintigraphy)}

\section{Methods and pitfalls}

Three-phase bone scintigraphy is the most widely used screening nuclear-imaging procedure for diagnosing prosthetic joint infections. It is most sensitive when the prosthesis has been implanted at least 2 to 5 years earlier (for hip and knee, respectively). Bone scintigraphy is largely available with low cost (Prandini et al., 2006). It consists of a dynamic imaging sequence (early phase), called the flow or perfusion phase (first phase), followed immediately by static images of the region of interest, the blood-pool or soft-tissue phase (second phase). The late images (third phase) consist of planar static images of the area of interest, acquired 2-4 $\mathrm{h}$ after the radiopharmaceutical injection with the possibility to perform a SPECT/CT for better anatomical localisation of lesions (Love et al., 2004). Radiopharmaceuticals used for bone scintigraphy are ${ }^{99 \mathrm{~m}} \mathrm{Tc}-$ labelled diphosphonates (MDP, HDP, DDP, HEDP), which are bone-seeking radiopharmaceuticals that selectively accumulate on the surface of bone mineral matrix, in areas of high metabolic activity. The accumulation of diphosphonates correlates with blood flow and with new bone apposition (Genant et al., 1974). Therefore, any cause of increased bone formation, e.g., physiological bone remodelling after prosthesis implantation or pathological conditions such as septic or aseptic prosthetic loosening, may show an intense peri-prosthetic activity on bone scan images (Love et al., 2001). Bone scintigraphy is therefore characterised by high sensitivity but low specificity for diagnosis of PJI.

\section{Interpretation criteria}

In the literature, discordant opinions about interpretation criteria of bone scintigraphy are mentioned. Some authors recommend a quantitative analysis while others support the use of a qualitative approach (Reinartz, 2009).

Several attempts have been made in the last decades to create a universally accepted classification that could help physicians interpret three-phase bone scan images. However, a solution has not yet been reached and probably will not be reached in the future also, due to the fact that the patterns of radiopharmaceutical uptake are extremely variable, also in asymptomatic patients, most obviously in patients with knee prostheses. Uptake after prosthetic surgery may be visible for years after the prosthesis placement. Several published trials have used profoundly different criteria. With regard to hip prosthesis, several authors accept the diagnostic criteria suggested by Wilson - who described three different patterns: (a) loosening of the stem with significant uptake in the area of the tip, in combination with at least a second substantial lesion in the lesser trochanter; (b) loosening of the acetabular cup, with continuous significant uptake in the cup-bone interface; and (c) infection, when late images and blood-pool images show a substantial uptake in prosthesis interfaces (Wilson et al., 1997). Nagoya et al. propose, for the diagnosis of infection, a positive finding in the early arterial phase while pathological uptake during the blood-pool phase, without increased perfusion, is suggestive of aseptic loosening (Nagoya et al., 2008).

It is universally accepted that a study with normal findings (no increased perfusion or blood-pool, no periprosthetic uptake in the late phase) can be considered as strong evidence against the presence of an infection. However, a positive bone scan with an increased vascularity - both in dynamic and in blood pool images and an increased metabolic uptake of ${ }^{99 \mathrm{~m}} \mathrm{Tc}$-diphosphonates in late images may indicate prosthetic joint infection; but as stated earlier, cannot correctly distinguish infections from other conditions such as post-surgical changes, aseptic loosening, or healing fractures. What should be kept in mind is that a bone scan may be positive for at least 2 years after hip prosthesis replacement and 5 years after knee prosthesis replacement due to physiological bone remodelling after implantation.

\section{Labelled leukocytes in vitro}

\section{${ }^{99 m}$ Tc-HMPAO and ${ }^{111}$ In labelled leukocytes: concept, pros and cons}

In vitro radiolabeling of autologous peripheral white blood cells (WBC) was already developed in the 1970s (Rennen et al., 2001). Labelled leukocytes do not accumulate at sites of increased bone turnover or remodelling in the absence of infection. The introduction of in vitro labelled $\mathrm{WBC}$ has improved the accuracy for the diagnosis of PJI (Love et al., 2009). Since neutrophils are predominantly labelled, and neutrophils are present in the infected prosthetic joint, WBC scintigraphy is nowadays the gold-standard radionuclide imaging technique for this purpose.

To have enough WBC to label, a blood sample of approximately $50 \mathrm{~mL}$ is collected and leukocytes are separated in vitro from red blood cells. These leukocytes are then labelled, either with ${ }^{99 \mathrm{~m}} \mathrm{Tc}-$ hexamethylpropylene amine oxime (HMPAO) or with ${ }^{111}$ In-oxine, and reinjected. Using the standard labelling procedures, only a few granulocytes are damaged by the labelling procedure, whereas most lymphocytes are killed. The damaged cells are rapidly cleared from the circulation after re-injection (Thakur and McAfee, 1984).

The use of HMPAO, a lipophilic chelator, allows for a high labelling efficiency of white blood cells with ${ }^{99 \mathrm{~m}} \mathrm{Tc}$. For most indications, because of the more optimal radiation characteristics, ${ }^{99 \mathrm{~m}} \mathrm{Tc}-$ labelled leukocytes are preferable. ${ }^{111}$ In has radiation energies of 171 and $245 \mathrm{keV}$, leading to a higher radiation burden, and a long half-life 
of $67 \mathrm{~h}$ that makes imaging possible only 24 or $48 \mathrm{~h}$ after injection. To compare: ${ }^{99 \mathrm{~m}} \mathrm{Tc}$ has a short half-life of $6 \mathrm{~h}$ and an ideal $\gamma$-radiation energy of $140 \mathrm{keV}$. On the other hand, a major advantage of ${ }^{111} \mathrm{In}$-leukocytes is that there is no major kidney, bladder or bowel excretion. In contrast, ${ }^{99 \mathrm{~m}}$ Tc-HMPAO is released with time from the labelled leukocytes, a process that starts within a few minutes after administration. Free ${ }^{99 \mathrm{~m}} \mathrm{Tc}-\mathrm{HMPAO}$ is excreted via the kidneys (within minutes) and by liver and gut (after several hours), thereby disturbing imaging of the abdomen after $3 \mathrm{~h}$. As a result, ${ }^{111} \mathrm{In}$-leukocyte imaging is preferred for evaluation of kidneys, bladder, gall bladder and intestine and may also be preferred if late images (after $24 \mathrm{~h}$ ) are needed, as in chronic infections (Peters, 1994). In all other indications, and especially in the evaluation of PJI, ${ }^{99 \mathrm{~m}} \mathrm{Tc}-$ HMPAO labelled leukocytes are the first choice (Larikka et al., 2001).

Autologous radiolabelled WBC have a high specificity, because they accumulate as a consequence of active migration into an infected tissue (Datz, 1994). They first adhere to vascular endothelium and then migrate towards the infected focus through endothelium and basal membrane.

Even if labelled leukocytes are the gold-standard nuclear method of choice for infection imaging, some limitations remain. Interpretation of WBC scintigraphy is complicated by the normal bio-distribution of the labelled WBC. They accumulate not only in infections, but also physiologically in the bone marrow (King et al., 1990; Palestro et al., 1992). To overcome this problem, imaging at several time points or combination of WBC scintigraphy with bone marrow scintigraphy may be performed and is already common in clinical practice.

The most important limitation is the laborious preparation of this radiopharmaceutical that must be performed in sterile conditions with a complicated and time-consuming procedure, which takes a trained technician around $3 \mathrm{~h}$. In addition, the need to handle potentially contaminated blood can result in hazard for technicians as well as for patients.

\section{Methods and pitfalls}

Although used for decades, labelling procedures, as well as image acquisition protocols and image interpretations may vary between different countries and even between different hospitals in the same country. Inconsistent results reported for WBC scintigraphy are related principally to differences in these protocols and interpretations (Tondeur et al., 2008). It is of utmost importance that these procedures and protocols are well standardised over the world. Recent published guidelines by the Infection Committee of the European Association of Nuclear Medicine (EANM) (De Vries et al., 2010; Roca et al., 2010) provide the indications, practical aspects, quality controls and safety procedures and should be followed around the world.

Interpretation of the images can be difficult even for expert physicians. The main interpretation difficulty is due to normal accumulation of WBC in the bone marrow, where the distribution may be extremely variable from one person to another. Labelled WBC accumulate in the bone marrow as a result of phagocytosis by the reticuloendothelial cells in the bone marrow. The distribution of the reticulo-endothelial component of the bone marrow closely parallels that of the haematopoietic component in most conditions; therefore, alterations in the distribution of the haematopoietic component are accompanied by similar alterations in the distribution of the reticulo-endothelial component (Palestro et al., 2006). The individual physiological variability of marrow distribution and its displacement in several different pathologic conditions could complicate the interpretation of WBC images because it makes it difficult to determine whether WBC uptake represents infection or active marrow. The migration of leukocytes and granulocytes in bone marrow also produces a lack of specificity in central bone because of a high percentage of "cold defects" due to lower perfusion of lessblood-supplied areas and existing necrotic bone. In addition to that, in response to systemic processes, such as various anaemias, tumours, and myeloproliferative diseases, there could be a generalised marrow expansion, which could be transient or permanent. In response to a local stimulus, such as fracture, inflammation or orthopaedic hardware, there could be a local marrow expansion, which is usually transient. In particular, haematopoietically active marrow usually develops around joint prostheses, producing an alteration of the normal distribution of the bone marrow. Furthermore, in peripheral bones, especially after orthopaedic surgery, there are often displacements of bone marrow with "hot spots" that must be distinguished from focal infection (Prandini et al., 2006).

In order to overcome the interpretation difficulties WBC scintigraphy can be associated with other imaging techniques: (1) three-phase bone scintigraphy, because leukocytes usually do not accumulate in sites of increased bone mineral turnover in absence of infection; and (2) bone marrow scintigraphy, because both the bone marrow radiopharmaceutical ${ }^{99 \mathrm{~m}} \mathrm{Tc}$-sulphur colloid and WBC accumulate in the marrow - while only leukocytes accumulate in infections. Furthermore, WBC images can be fused with CT or MRI images, of the part of the body suspected to have infection or inflammation, for a more accurate localisation of WBC uptake - particularly in differentiating soft tissue uptake from bone uptake.

\section{Interpretation criteria}

Accumulation of labelled WBC in infection sites is a dynamic process, depending on preserved chemotaxis and cellular response in a given process (Signore and Glaudemans, 2011). Accurate interpretation of labelled WBC scintigraphy requires knowledge of the normal (blood and bone marrow) and abnormal variants of WBC localisations in different tissues and organs (Fig. 2) and the variation in time in different infectious and inflammatory conditions.

The diagnosis of PJI is made by qualitative or semiquantitative comparison of delayed (after 3-4 h) versus late images (after 20-24 h). The images are visually classified as: (a) negative if no uptake or a significant decrease in uptake from delayed to late images is present; (b) positive when uptake is seen in both delayed and late images with increase of activity or size in time; and, (c) equivocal when 
Fig. 2. Normal (blood and bone marrow) and abnormal time variations of WBC localisations with time. This graph gives the basis for three timepoint imaging using radiolabelled WBC for discriminating between infection and sterile inflammation and bone marrow activity. As can be seen in the figure, uptake in the bone marrow is high at an early time point (1 h) and remains stable. Infection (acute, subacute and chronic) show increase in uptake with time, whereas sterile inflammation shows uptake but decreases in time.

Fig. 3. Three phase bone scan with ${ }^{99 m}$ Tc-MDP (A and B) and dual time point imaging with radiolabelled autologous WBC (C and $\mathbf{D})$ in a patient 1 year after a hip joint replacement on the left side. (A) Blood pool image with increased perfusion around the prosthesis, (B) late static image anterior view with increased uptake around the prosthesis. This bone scan could be the result of the recent surgery, loosening of the prosthesis or an infection. To differentiate, a leukocyte scan was performed: (C) delayed image anterior view (4 h) without uptake, and (D) late image anterior view (24 h) with uptake around the prosthesis and at the tip of the prosthesis, clearly indicating the infection.
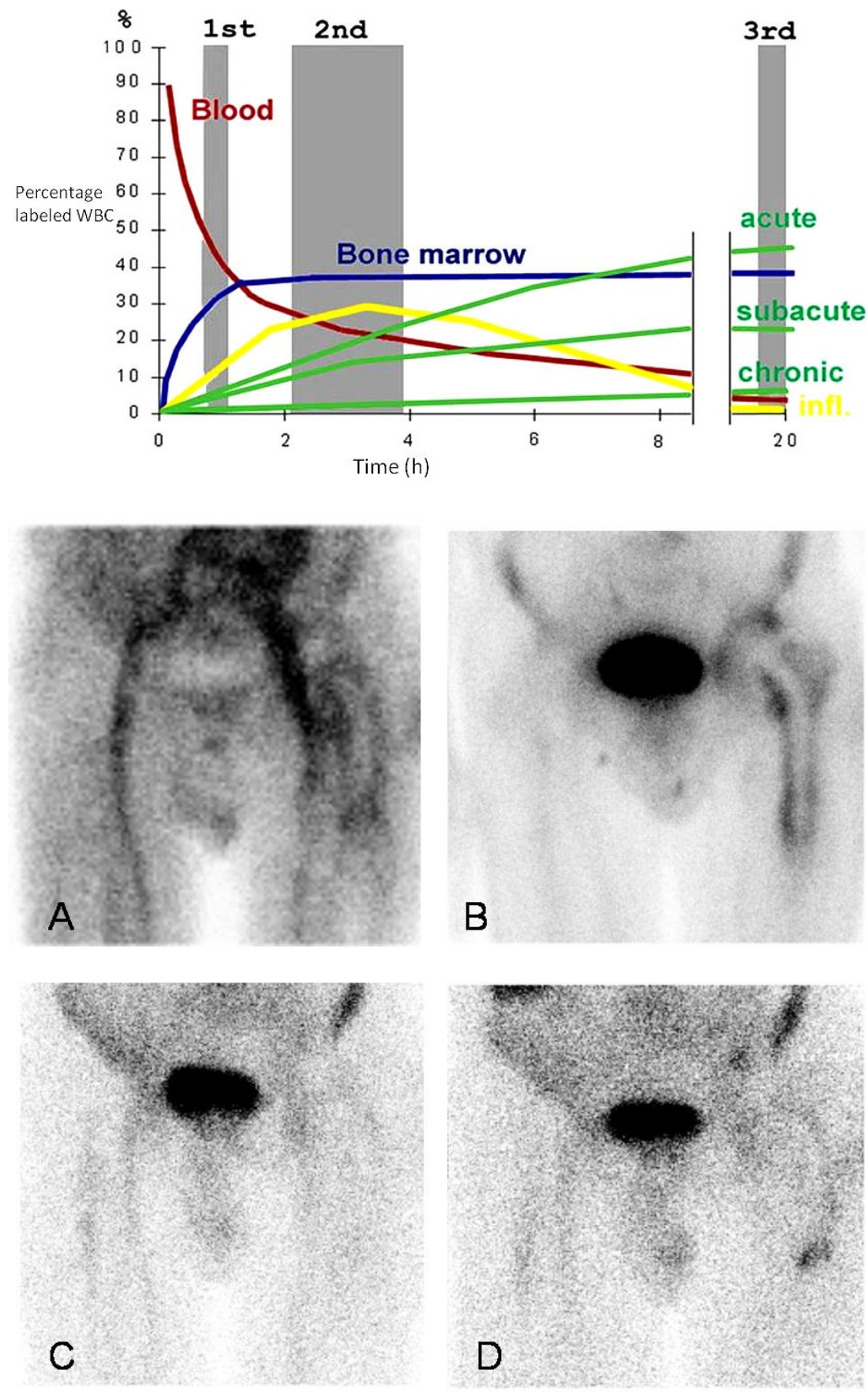

uptake in delayed and late images is the same or slightly decreasing. This classification is illustrated in Fig. 3A-D.

This qualitative analysis may be operator-dependent if the delayed and late images are elaborated or reprocessed in different ways, or with different contrast and background. It has therefore been suggested that images should be equalised, using bone marrow activity as a reference. This method has the great limitation that the assumption is that bone marrow activity does not change with time, which is true in most cases but false in cases of bone marrow stimulation by therapies, prosthetic devices or just postural forces. We therefore suggested acquiring images with different acquisition times, corrected for isotope decay, and to normalise all images with the same contrast and background so that early, delayed and late images may have the same counting statistics and can be compared without operator bias (Familiari et al., 2011).

After visual or qualitative assessment, semi-quantitative analysis may also be performed to help differentiating between infection and non-specific uptake in order to evaluate the activity of labelled WBC around the symptomatic prosthesis. Regions of interest (ROIs) can be drawn over the part of the prosthesis suspected of being infected and compared with presumed normal reference tissue (e.g., anterior-superior iliac crest, unaffected contralateral bone, etc.). The mean counts per pixel in these ROIs are recorded to calculate Lesion-to-Reference (L/R) ratios both in delayed and late images. When the $\mathrm{L} / \mathrm{R}$ ratio decreases with time the examination is classified negative for infection, but when the $\mathrm{L} / \mathrm{R}$ ratio increases with time 
the scan is considered positive for infection and when the $\mathrm{L} / \mathrm{R}$ ratio is similar or slightly decreases with time the scan is considered equivocal.

If SPECT/CT images are used, the delineation of the site of increased uptake may be calculated by a $50 \%$ iso-contour on a single trans-axial slice with the hottest activity and the reference tissue. Hybrid (SPECT/CT) imaging is mandatory in case of doubtful planar images. The same criteria as for planar images may be used for semi-quantification.

In 2004, Pelosi et al. studied 78 patients with suspected hip or knee prosthetic infection using ${ }^{99 \mathrm{~m}} \mathrm{Tc}$-labelled WBC and showed that the addition of a quantitative evaluation to the qualitative analysis of delayed and late images leads to a significant improvement in both specificity and accuracy of the method (Pelosi et al., 2004). In 2007, Ballani et al. evaluated in 24 patients, 7 of whom with hip or knee arthroplasty, the value of semi-quantitative uptake measurements of ${ }^{99 \mathrm{~m}} \mathrm{Tc}-\mathrm{MDP}$ and ${ }^{99 \mathrm{~m}} \mathrm{Tc}-\mathrm{HMPAO}-\mathrm{WBC}$ comparing the degree of the two radiopharmaceutical uptake in the area of suspected infection. The semiquantitative measurement and thus the comparison of the two images has been made on anterior images obtained $3 \mathrm{~h}$ after MDP injection and on images obtained only $4 \mathrm{~h}$ after WBC injection. The study concluded that the bone scan/labelled WBC association is most useful in excluding infections when the findings are clearly negative (Ballani et al., 2007).

As mentioned earlier, sometimes it is useful to have a combined labelled WBC-bone marrow imaging in order to discriminate, if a high uptake is due to the presence of infection or to displacement of activated bone marrow. This is particularly helpful in cases where quantification does not help (equivocal cases). Bone marrow imaging can be performed with ${ }^{99 \mathrm{~m}} \mathrm{Tc}$-sulphur colloid (most often used in the United States) or with ${ }^{99 \mathrm{~m}} \mathrm{Tc}$-nanocolloid (most often used in Europe). Nanocolloid has smaller particles, thereby showing more uptake in the bone marrow compared to sulphur colloid. The differential diagnosis is made visually by comparing uptake of the bone marrow agent with the uptake of the labelled leukocytes. When there is activity on the WBC images without corresponding activity on the bone marrow images, the leukocyte uptake is due to infection; when the distribution of the two radiopharmaceuticals is similar or spatially congruent, the labelled leukocyte activity is due to uptake in bone marrow (Love et al., 2001). At present, a subjective visual assessment of the different distribution of the two radiopharmaceuticals is the only clinically used method. An absolute quantification of labelled WBC uptake, in the peri-prosthetic area and in normal bone marrow regions, is difficult because the images are affected by many factors, e.g., camera configuration, patient size, position of the patient, other individual characteristics etc. Therefore, a relative, rather than absolute, quantification can be clinically more relevant ( $\mathrm{Zhu}$ et al., 2007). It is worth mentioning that, in this study the WBC labelling took place with ${ }^{111}$ In-oxine-WBC and bone marrow labelling with ${ }^{99 \mathrm{~m} T c}$-colloids. Maybe there are more quantitative possibilities when using the same radionuclide. A last method is to use dual-time WBC scintigraphy with early
( $1 \mathrm{~h}$ after injection) imaging as a surrogate for bone marrow uptake. Subsequently, incongruence between early and late images, analogous to conventional WBC scintigraphy and bone marrow scintigraphy, is indicative (Pelosi et al., 2004).

\section{Closed systems to label leukocytes in vitro}

To avoid the afore-mentioned disadvantages of the labelling procedure of autologous leukocytes, simplified labelling procedures providing protection for technicians and patients and the possibility to apply the procedure simultaneously to several patients have been developed. Recently, two sterile single-use closed devices for introduction, handling, labelling and withdrawal of solvents, liquids and cells under sterile conditions - and without changing internal pressures - were invented and patented (Signore et al., 2012). These devices improve the quality and ease of the whole labelling procedure, offer high level of protection to operator and patients, are safe and easy to use, and make this labelling technique also available for more clinical centres and more patients. By using these devices it could also force centres to work with the same procedure.

\section{${ }^{18}$ F-FDG labelled leukocytes: concept, pros and cons} Leukocytes have also been labelled in vitro with ${ }^{18}$ F-fluorodeoxyglucose (FDG), in an attempt to develop a more specific, positron-emitting radiopharmaceutical for PET or PET/CT imaging. The first results of initial clinical investigations were encouraging (Dumarey et al., 2006; Rini and Palestro, 2006; Rini et al., 2006) and whole body and organ dosimetry were comparable with results of ${ }^{111} \mathrm{In}$-labelled leukocytes (Forstrom et al., 2002). However, several limitations were found for this procedure. The labelling efficiency of leukocytes with ${ }^{18} \mathrm{~F}-\mathrm{FDG}$ is significantly less than that for ${ }^{99 \mathrm{~m}} \mathrm{Tc}-\mathrm{HMPAO}$ or ${ }^{111}$ In-oxine, because blood glucose levels and metabolic status of cells affect this. Most importantly, the half-life of ${ }^{18} \mathrm{~F}$ is only $110 \mathrm{~min}$. Consequently, because of the low labelling efficiency and the short half-life, the labelling process must be started with two or three times the amount of radioactivity that will eventually be injected into the patients (Palestro et al., 2007). It also means that it is technically not feasible to perform imaging much later than 4-6 h after injection. In most clinical situations, and for sure in PJI, imaging at late time points (20-24 h post injection) is mandatory because of the slow leukocyte accumulation at infected sites as compared to bone marrow. Such a delay between injection and imaging is not possible when using ${ }^{18} \mathrm{~F}$ as label.

\section{Labelled leukocytes in vivo}

Labelled monoclonal antibodies: concept, pros and cons Considerable effort has been devoted to develop in vivo methods for leukocyte labelling that could eliminate the limitations of in vitro labelled leukocytes and that could increase sensitivity and specificity. Most efforts have been made in investigating monoclonal antibodies (MoAbs) against specific surface receptors on granulocytes. 
Granulocytes express a variety of surface antigens that could be targeted for binding with a suitable antibody or fragment. Those antigens are expressed in activated granulocytes during inflammatory processes. The use of these radiolabelled MoAbs has the advantage that it is easier, compared to the use of radiolabelled autologous leukocytes and does not need the handling of potentially hazardous biological specimens. Disadvantages, however, are their high molecular weight (resulting in slow diffusion into sites of inflammation), a long plasma half-life, low concentration at the affected site and high uptake in the liver and bone marrow, due to clearance by the reticuloendothelial system. A long interval is therefore required between the administration of labelled MoAbs and the acquisition of images in order to have a good target-tobackground ratio (6-24 h). Furthermore, only a minor percentage of the injected antibody really binds directly to the cells. Most of it will concentrate in the inflamed areas, due to non-specific leakage as a result of increased permeability - independently of whether there is an infection or just a sterile inflammation.

The use of antibody fragments (Fab') of human antibodies could overcome the limitations of complete antibodies; theoretically, immunogenicity is lower, blood clearance is faster and accumulation in infected areas is higher. However, the theoretical advantage of a higher accumulation in infected areas is also considered a limitation because of the high non-specific leakage in sterile but inflamed tissues as in loosening of prostheses.

Radiolabelled MoAbs or their fragments are always injected in a tracer (non-pharmacological) dose for scintigraphic imaging, which rarely induces any clinical or side effects in patients. One of the side effects that has been described in some cases of repeated murine MoAb administration is the induction of human murine antibodies (HAMA), a host response to foreign antibodies. If MoAbs are injected in patients with HAMA, their distribution may be affected, as well as image quality and clinical relevance of images.

The commercially available ${ }^{99 \mathrm{~m}} \mathrm{Tc}-$ labelled antigranulocyte IgG1 murine antibody BW 250/183 (Besilesomab, Scintimun $^{\circledR}$, molecular weight $150 \mathrm{kDa}$ ) binds to the cross-reacting antigen 95 (NCA-95), expressed on human granulocytes. This antigen is non-specific, because it is also expressed on the epithelium of the lung, in the colon and on macrophages (Becker et al., 1994; Kumar, 2005). The possibility of inducing HAMA, particularly in patients receiving repeated injections, is a limitation of the use of this radiopharmaceutical in follow-up studies (Becker and Meller, 2001).

Another murine MoAb, anti-stage specific embryogen antigen-1 (Anti-SSEA-1, molecular weight $900 \mathrm{kDa}$ ), called ${ }^{99 m}$ Tc-fanolesomab $\left(\right.$ LeuTech $\left.^{\circledR}\right)$, binds with high affinity to the CD15 antigen expressed on neutrophils, eosinophils and lymphocytes. The antibody binds to neutrophils in greater proportion than to any other cell type (Love and Palestro, 2003). It can be used at a low dose, thereby not inducing HAMA. The in vivo binding on polymorphonuclear cells exceeds $50 \%$, suggesting involvement of more specific accumulation in infected sites, such as in vivo migration of leukocytes from the circulation to the focus (Rennen et al., 2001). In 2005 it was withdrawn from the market because of reports of serious and life-threatening cardiopulmonary events; the precise explanation of these events has yet to be elucidated (Palestro et al., 2007).

${ }^{99 \mathrm{~m}}$ Tc-sulesomab (LeukoScan ${ }^{\circledR}$ ) is a murine $\operatorname{IgG} 1$ antibody Fab' fragment (molecular weight $90 \mathrm{kDa}$ ) that binds to NCA-90 on granulocytes. It does not induce HAMA response andextravasates into infected sites non-specifically by increased vascular permeability. Nevertheless, it may be accumulating after local binding to primed granulocytes or binding to activated migrated extravascular granulocytes (Skehan et al., 2003).

\section{Methods and pitfalls}

The uptake mechanism of radiolabelled MoAbs is probably twofold: migration of targeted cells to the infectious focus due to chemotaxis, but also non-specific uptake of free antibody due to an increased capillary permeability at the infectious focus, with subsequent binding to granulocytes (Skehan et al., 2003). About $10 \%$ of the injected activity is bound to the granulocytes at 45 min after injection, and $20 \%$ of the activity is circulating freely in the blood.

NCA-95, the antigen to which Scintimun ${ }^{\circledR}$ is directed, is found on granulocytes and mature bone marrow cells of the granulocytic lineage. These cells are present in major amounts in infectious lesions, but are also found in the haematopoietic bone marrow. The typical uptake patterns of anti-granulocyte MoAbs, therefore, includes focal uptake at sites of infection as well as staining of the haematopoietic bone marrow (Richter et al., 2011).

\section{Interpretation criteria}

The same concepts as described for WBC scintigraphy can be applied for the use of anti-granulocyte MoAbs. Thus, early, delayed, and late images (30 min, 3-4 h and 20-24 h) should be acquired and SPECT/CT images provide a useful benefit for the localisation of infections, being mandatory in some circumstances. Late images at 20-24 h have been recommended to increase sensitivity (Palestro et al., 2007). The image acquisition criteria and the image interpretation criteria are similar to that reported for WBC but a few considerations must be made. At first, radiolabelled antibodies do bind more to the bone marrow than radiolabelled WBC, thereby making the use of bone marrow scintigraphy as a reference background difficult. Secondly, the anti-granulocyte monoclonal antibodies bind to circulating granulocytes (that will later migrate to infection site) but also to tissue infiltrating granulocytes, thus an infection site can be positive also at delayed time points (3-4 h) as well as late (20-24 h) time points. Moreover, antibodies, having a small molecular weight (antibody fragments), may accumulate in sites of sterile inflammation due to extravascular leaking with oedema, thus giving false positive results. Nevertheless, results of recently published studies in patients with PJI show the high diagnostic accuracy of radiolabelled antibodies (besilesomab and sulesomab), similarly to radiolabelled WBC (Iyengar et al., 2006; Blazeski et al., 2010; Richter et al., 2011; Sousa et al., 2011). Another study that compared an intact MoAb (besilesomab) with a fragmented MoAb 
(sulesomab) found similar diagnostic accuracy rates for the detection of septically loosened total knee arthroplasty (Gratz et al., 2012).

\section{${ }^{18}$ F-Fluorodeoxyglucose: concept, pros and cons}

${ }^{18} \mathrm{~F}-\mathrm{FDG}$ is a radiopharmaceutical for PET imaging, used for many years in oncology, but it can also be used in infectious diseases because activated leukocytes use glucose as an energy source after activation. Its accumulation and retention in leukocytes depends on the activation/metabolic status of these cells and on the ratio between phosphatase and hexokinase. Phosphatase is able to phosphorylate FDG, thus avoiding its further metabolism and efflux from the cells - but, hexokinases can revert the phosphorylation and cause FDG efflux from cells. The accumulation of FDG in leukocytes is therefore still poorly understood. However, it is being exploited more and more for the imaging of infections. In fact, ${ }^{18} \mathrm{~F}$-FDG-PET is now increasingly replacing the conventional scintigraphic methods.

\section{Methods and pitfalls}

As for any other nuclear medicine imaging technique, when interpreting a ${ }^{18} \mathrm{~F}$-FDG-PET scan the nuclear medicine physician is supposed to be familiar with the physiological distribution of the radiopharmaceutical used in order to be able to discriminate between physiological and pathological uptake. Physiological uptake of FDG includes brain, heart, kidneys, bladder and sometimes intestines. To minimise FDG uptake in normal tissue, patients must fast for at least several hours to reduce competition for glucose transporters. After the injection, patients must rest for an hour and limit physical activity to minimise muscle uptake of FDG. High-contrast images of infectious lesions can be obtained early (60 min) after radiopharmaceutical injection.

No physiological uptake should be seen in the bone itself, but - especially in patients with infections or inflammation - the bone marrow, at various levels, can show increased uptake. In PJI, however, the problem is the generation of artefacts, characterised by artificial FDG uptake adjacent to prostheses (Goerres et al., 2003). Furthermore, non-specific FDG uptake may be seen in healing tissues, up to 6 months after surgical intervention (Zhuang et al., 2003; Jones-Jackson et al., 2005;), bone fractures and atherosclerotic lesions. A negative FDG study may spare revision surgery, but a positive scan must be interpreted with caution since long-lasting nonspecific increased uptake may be responsible for false positive studies (Israel and Keidar, 2011).

\section{Interpretation criteria}

The same difficulties as described for three-phase bone scans in finding universally accepted interpretation criteria are present for FDG-PET imaging. Evaluation of images can be done both qualitatively and semi-quantitatively. For a qualitative analysis, PET images are visually analysed for increased FDG uptake, considering pattern (focal, linear, and diffuse), intensity and relationship to areas of physiological distribution (Fig. 4). The physiological findings by FDG-PET can be compared to morphological

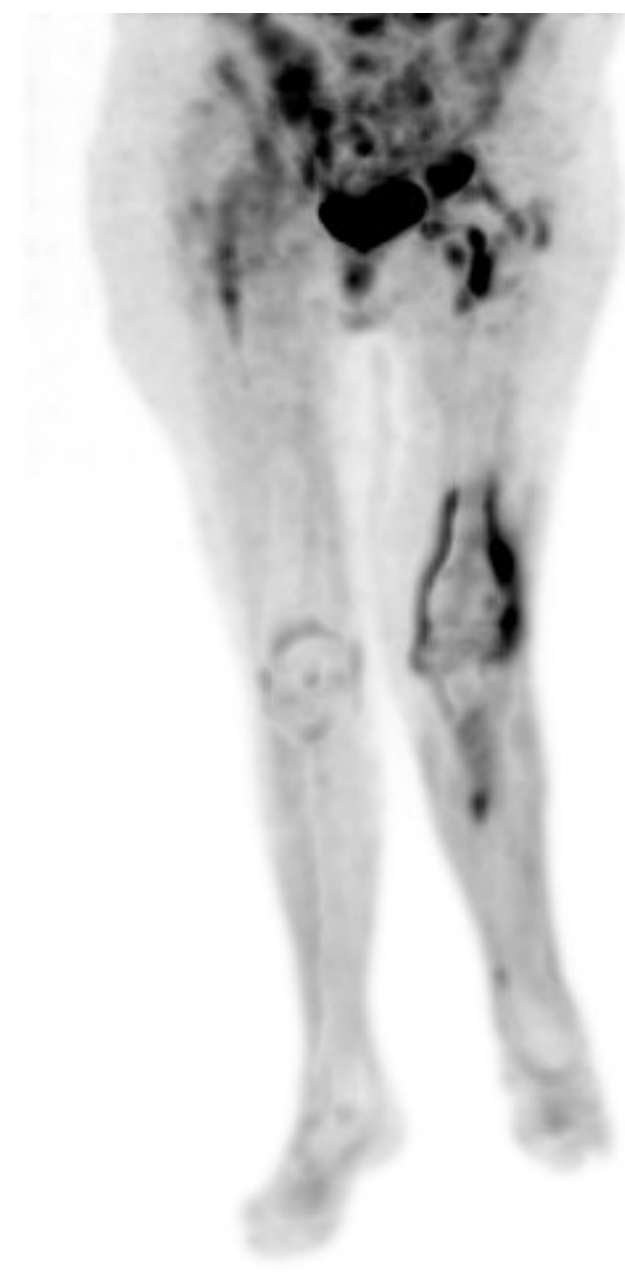

Fig. 4. ${ }^{18}$ F-FDG-PET scan of a patient with left hip joint prosthesis and knee joint prosthesis. Pain complaints since several months and elevated CRP and ESR. FDGPET revealed high uptake in the cup and trochanter region of the hip prosthesis and high uptake around the knee prosthesis, both suspicious for infection. Note also the uptake in the bone marrow of the proximal femur on the right side, which is compatible with an infection.

information obtained by CT (Fig. 5). It must be kept in mind that the sensitivity to detect infection is not absolute and therefore, even with a negative PET, a thorough reading of the CT slices is essential.

In the literature, five different patterns of PET findings in prosthetic joint replacements are described, based on a qualitative visual analysis of FDG distribution. Three patterns (no increased uptake, uptake around the prosthesis neck, and uptake around the neck accompanied by uptake around parts of the cup and/or around the proximal shaft) indicate no loosening. Increased uptake in the neck of the prosthesis, accompanied by uptake around the whole cup of the prosthesis and/or around the whole shaft, fits with aseptic prosthetic loosening. Finally, infection is characterised by increased uptake in all the abovementioned prosthesis-bone interfaces - including periprosthetic soft tissues (Reinartz et al., 2005). Nevertheless, these criteria have not been accepted by all, and are also criticised. 


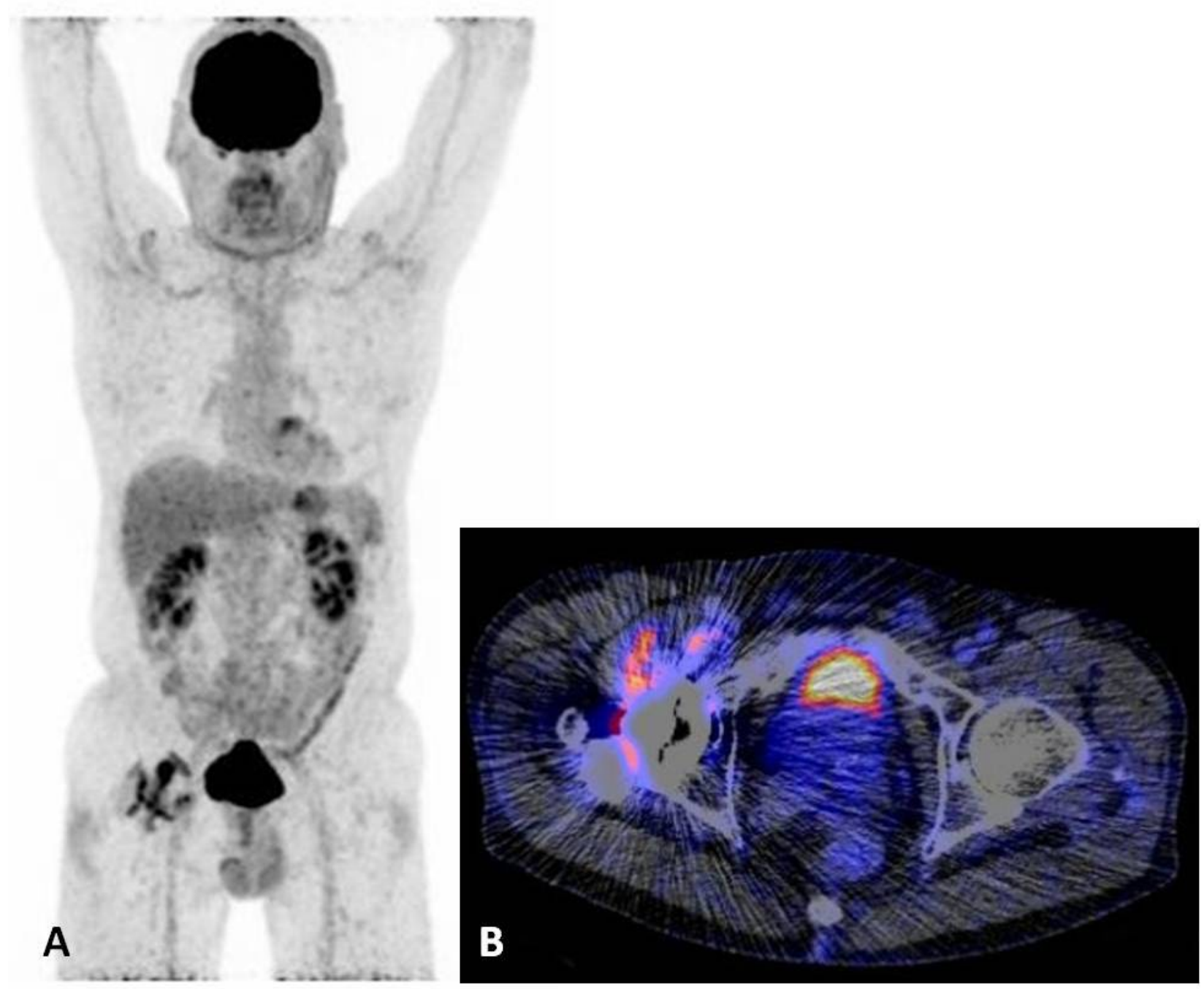

Fig. 5. ${ }^{18}$ F-FDG PET coronal view (A) and PET-CT transaxial slice, fusion image (B) of a patient with an infected hip prosthesis on the right side. Notice in (A) the physiological uptake in the brain, the left ventricle, the liver, kidneys and bladder. There is a pathologically increased uptake around the hip prosthesis on the right side and within the bone. This is highly suggestive for an osteomyelitis associated with soft tissue infection.

For a semi-quantitative analysis, Standardised Uptake Value (SUV) should be taken into consideration even though its use has not been yet validated in inflammation and infection as it is in oncology. Attempts made to use it in the discrimination between inflammation and infection have still been in vain. Therefore, calculation of the SUV should be used with caution in this field.

In a study of 59 patients with failed hip or knee arthroplasty, FDG-PET with the combined use of labelled leukocyte scintigraphy and bone marrow scintigraphy were investigated. PET images were interpreted by using 4 different criteria (1: any periprosthetic activity, 2: periprosthetic activity on the FDG image, without corresponding activity on the bone marrow image, 3 : only bone-prosthesis interface activity, 4: semi-quantitative analysis). Regardless of how the images were interpreted, FDG-PET imaging was found to be less accurate than labelled WBC/bone marrow imaging for diagnosing PJI (Love et al., 2004).

\section{In vivo imaging of bacteria}

Concept, pros and cons

As mentioned, radionuclide imaging has been extensively used for infection imaging using in vitro and in vivo labelled leukocytes. A totally different strategy is to target directly the responsible micro-organisms in vivo, without intervening leukocytes. This strategy is used for developing new radiopharmaceuticals that couple SPECT or PET isotopes with various antibiotics, vitamins, bacteriophages and antimicrobial peptides. These radiopharmaceuticals enable diagnosis of the infection and may also be used to monitor anti-inflammatory therapies. However, since the main obstacle to imaging PJI is the biofilm generated by bacteria, it seems that these molecules are unable to cross the biofilm and bind to target bacteria. By contrast, even if growing under a biofilm, bacteria still secrete chemotactic factors for leukocytes; thus, using radiolabelled WBC, it could still be possible to image leukocyte migration in $\mathrm{PJI}$ in the presence of biofilm. These aspects have never 


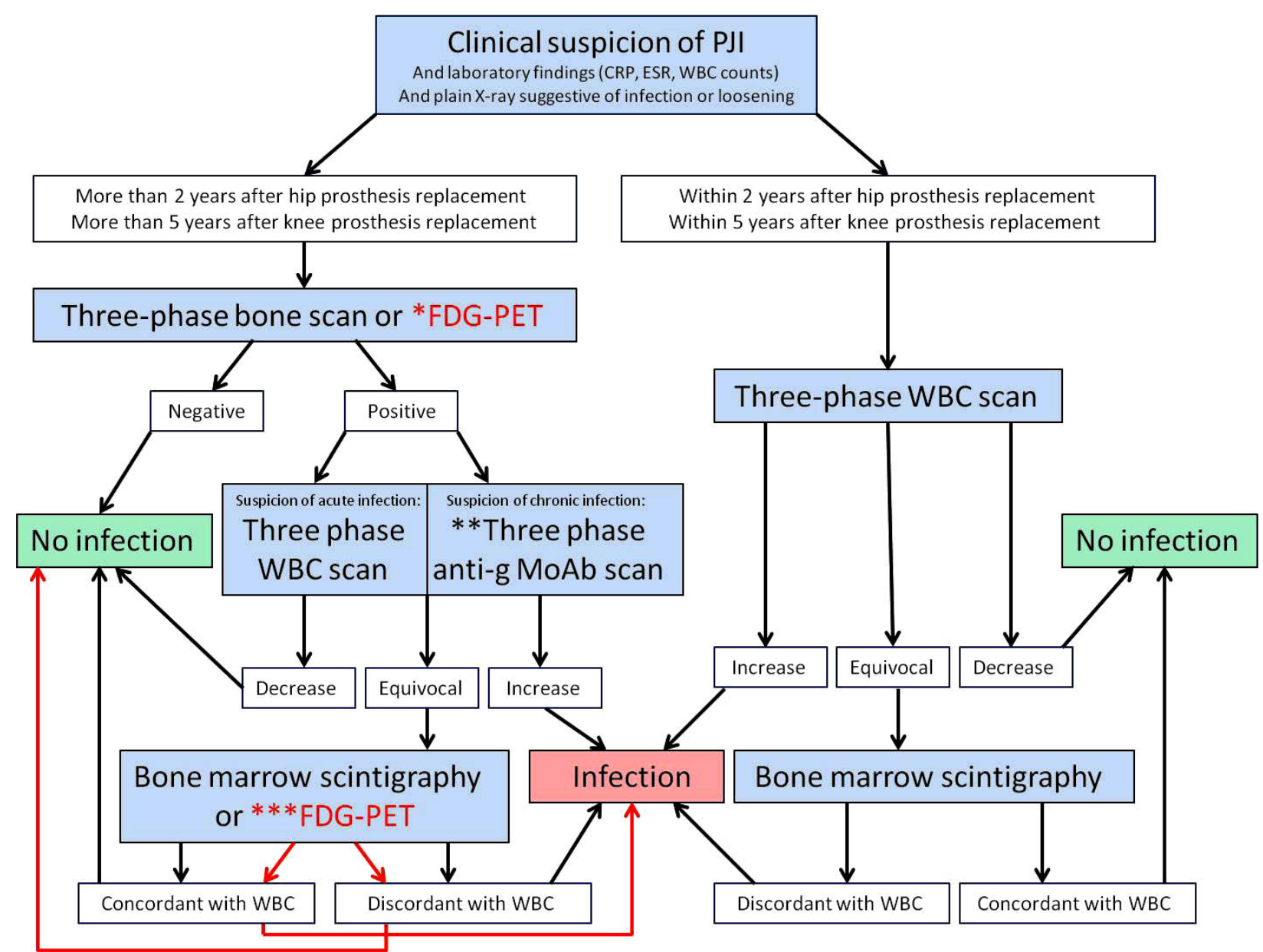

Fig. 6. Schematic diagnostic pathway for imaging PJI. There is a different pathway depending on the age of prosthesis and the probability of infection. *An FDG-PET scan can be performed in patients with low probability of infection (normal WBC counts, or ESR, or CRP) due to the high sensitivity of this technique. **In patients with a suspected chronic infection the scan with anti-granulocyte MoAb has been reported to be slightly more accurate than WBC scan. ***In patients with an equivocal WBC or anti-granulocyte scan, particularly if SPECT-CT is not available or doubt consists in differential diagnosis between osteomyelitis and soft tissue infection, FDG-PET may be useful due to its high special resolution and sensitivity. By contrast, if differential diagnosis is between osteomyelitis and bone marrow expansion, the gold-standard technique is to combine a bone marrow scan with radiolabelled colloids.

$\mathrm{PJI}=$ prosthetic joint infection, $\mathrm{CRP}=\mathrm{C}$-reactive protein, $\mathrm{ESR}=$ erythrocyte sedimentation rate, $\mathrm{WBC}=$ white blood cells, anti-g = anti-granulocyte, $\mathrm{MoAb}=$ monoclonal antibody, FDG-PET = fluorodeoxyglucose-positron emission tomography.

been carefully investigated in vitro or in vivo and the above consideration is rather speculative. We will now describe several radiopharmaceuticals that target directly bacteria and that are potentially useful for diagnosing PJI. However, at this moment, they have not been tested on a large population for this indication.

\section{Radiolabelled antibiotics}

The first described labelled antibiotic for infection imaging was ciprofloxacin (Britton et al., 2002). This is a broad-spectrum antibiotic, taken up by bacteria but not by the mammalian cells. Once internalised, it binds to the DNA-gyrase and topoisomerase IV and is unable to leave the bacterial cell. Therefore, ${ }^{99 \mathrm{~m}} \mathrm{Tc}$-ciprofloxacin (Infecton ${ }^{\circledR}$ ) could be used to identify live bacterial infection (Vinjamuri et al., 1996). Infecton ${ }^{\circledR}$ is mainly excreted via the kidneys, it has low liver metabolism and bowel uptake is usually low. At first, it was proposed as an agent for distinguishing sterile inflammation from bacterial infection. However, since its introduction in 1993, this has never been confirmed (Gemmel et al., 2009). It was shown to be a sensitive and quite specific marker of infection in a large multicentre study coordinated by the International Atomic Energy Agency (Britton et al., 2002), but this high specificity was not found in another paper (Dumarey et al., 2002). Possible explanations for these contradicting results include the presence of ciprofloxacin-resistant bacteria, biofilm, insufficient numbers of viable intra-lesional bacteria and the use of similar antibiotic therapy before imaging (Alexander et al., 2005). It was also reported that the discrepancy in the ciprofloxacin performance was due to different kit formulations for the radio-ciprofloxacin 


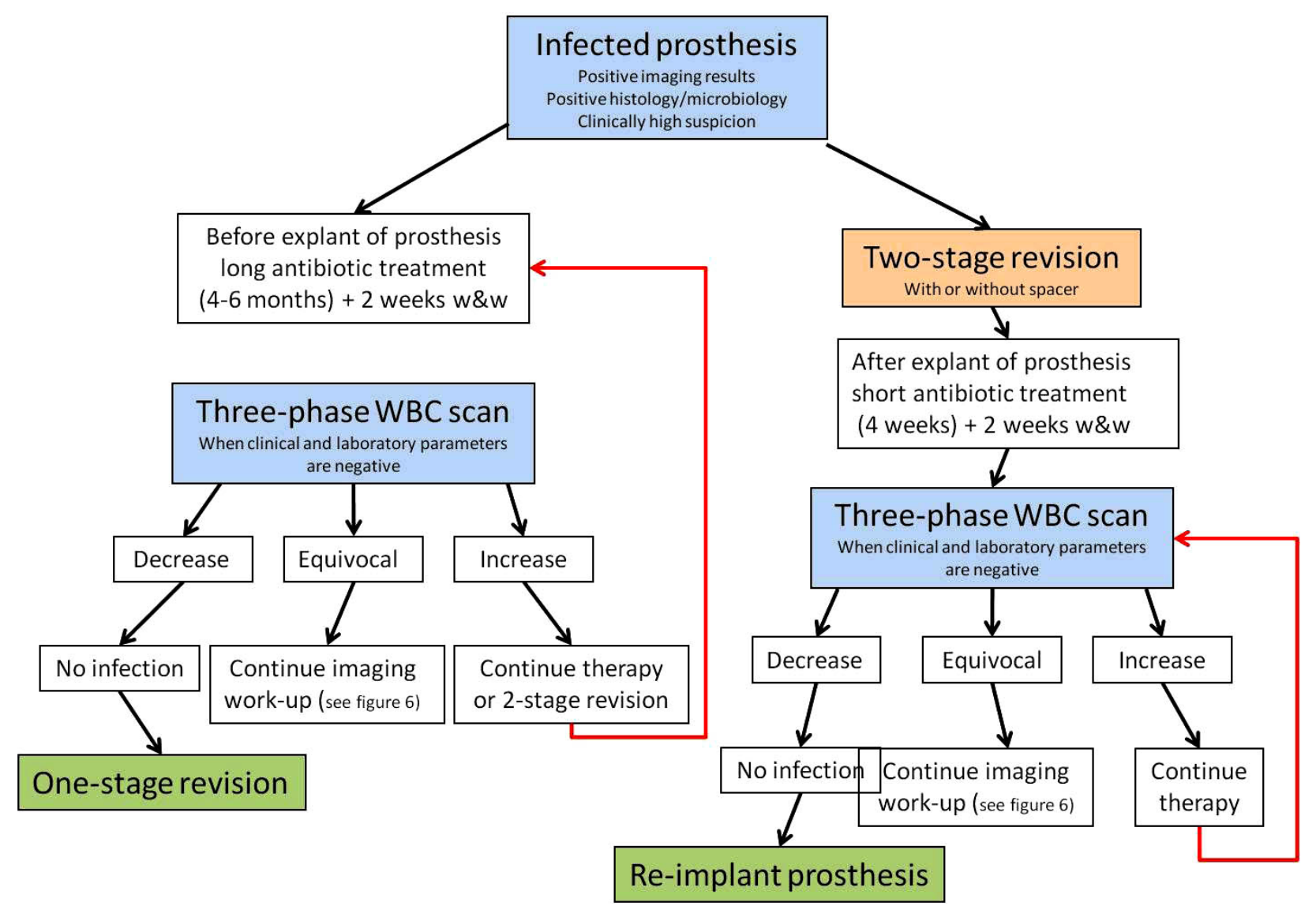

Fig. 7. Proposed diagnostic flow chart for the proven infected prosthesis. In this proposal, WBC scintigraphy is used in the flow diagram to support decision-making (continue or stop antibiotic treatment, time-point for re-implantation). $\mathrm{w} \& \mathrm{w}=$ wait and watch, $\mathrm{WBC}=$ white blood cells.

preparation used in different studies (Britton et al., 2004). Overall, at the moment, there is no convincing evidence of its efficacy.

Several other antibiotics have been radiolabelled with ${ }^{99 \mathrm{~m}} \mathrm{Tc}$, most of them members of the quinolone antibiotics, some of them also with ${ }^{18} \mathrm{~F}$ for PET imaging. Preliminary studies have shown significantly in vitro uptake in bacteria as well as accumulation at sterile inflammatory sites. However, critical evaluation of these compounds under investigation is warranted, ultimately to prove their value for specific infection imaging (Gemmel et al., 2009; Gemmel et al., 2012).

\section{Radiolabelled vitamins}

Avidins are a family of proteins present in the eggs of avians, amphibians and reptiles. Biotin (vitamin $\mathrm{H}$ ) is a water-soluble vitamin of the B-complex group of vitamins, has a low molecular weight $(224 \mathrm{Da})$, and is a growth factor for the majority of bacteria. In particular, pyruvate carboxylase, a key metabolic pathway for obtaining energy by ATP cleavage, is biotin-dependent, and bacterial acetylcoA carboxylase is a biotin-dependent enzyme utilised in the first step of fatty acid syntheses (Attwood, 1995; Yao et al., 1997). Infection imaging with avidin $/{ }^{111}$ In-biotin is based on non-specific accumulation of unlabelled avidin at sites of infection due to locally enhanced vascular permeability, followed approximately $4 \mathrm{~h}$ later by intravenous injection of the labelled biotin. Advantages of this method are a good target-to-background ratio and early imaging (Rusckowski et al., 1992). The excretion is rapidly via the kidneys with minimal uptake by normal tissues.

The labelling of biotin with ${ }^{111} \mathrm{In}$ is an easy procedure and shows high efficiency and stability (>98\% until $24 \mathrm{~h}$ ). The main advantages are the absence of uptake of healthy bone marrow of the spine, and the possibility to study the patients in follow-up during antibiotic treatment without any suspension of the therapy (Lazzeri et al., 2008; Lazzeri et al., 2010). Limitations are false-positive results (rarely) when a large inflammatory process leads to leakage of the radiopharmaceutical, because of altered capillary permeability, and false-negative results when microorganisms that do not utilise biotin for their own growth cause the infective pathologies. The complete urinary excretion of ${ }^{111} \mathrm{In}$ results in a relatively high dosimetry for the kidneys.

\section{Radiolabelled bacteriophages}

Another application of a potential infection-specific agent is the introduction of radiolabelled bacteriophages, which are highly specific for bacteria. Bacteriophages are viruses that show no specificity for mammalian cells and infect bacteria exclusively (Rusckowski et al., 2004). 
The binding mechanism consists of the attachment of the bacteriophages to specific surface receptors located on the surface of the bacteria.

Because bacteriophages have a natural specificity for bacteria, it is possible to develop radiolabelled bacteriophages as infection-specific agents. The M13 bacteriophage was labelled with ${ }^{99 \mathrm{~m}} \mathrm{Tc}$ and a rapid uptake in mouse models as well as in bacterial strains in vitro was found (Rusckowksi et al., 2004). Several radiolabelled bacteriophages were recently further investigated as potential agents for specific imaging of infection, with only one showing specificity for its host bacteria (Rusckowski et al., 2008).

\section{Antimicrobial peptides}

Antimicrobial peptides play a critical role in the innate immune system, providing protection against microbial attacks. They are produced by various types of cells such as phagocytes, endothelial cells and many others. Their expression is induced upon contact with microorganisms or microbial products, like lipopolysaccharides or proinflammatory cytokines. The basis of their antimicrobial activity is the direct interaction with the bacterial plasma membrane by electrostatic and hydrophobic interaction (Chianelli et al., 2008). A range of human antimicrobial peptides, e.g., human lactoferrin (hLF), ubiquicidin 29-41 peptide fragment (UBI 29-41), human neutrophil peptide 1-3 (HNP 1-3), bacteriophage and synthetic peptides derived from these natural peptides, have been investigated as radiopharmaceuticals for imaging of infections (Akhtar et al., 2012).

${ }^{99 m}$ Tc-UBI 29-41, radiolabelled synthetic fragment of ubiquicidin, is an antimicrobial peptide that preferentially binds to bacteria and fungi in vitro and accumulates at the site of infection in experimental animal studies, with fast renal clearance with minimal hepatobiliary excretion and the ability to detect infection foci in humans. The agent cleared rapidly from the body, but the uptake in the infectious lesions and target-to-background ratio was low (Melendez-Alafort et al., 2004). Several encouraging studies on the usefulness of ${ }^{99 \mathrm{~m}}$ Tc-UBI 29-41 are on-going at present but data are still limited (Baldoni, 2009).

HNP 1-3 is a member of the $\alpha$-defensins, a potent immune adjuvant inducing the production of lymphokines, which promote $T$ cell and antigen-specific body production. It shows a higher binding to bacteria than to activated leukocytes. The peptide is rapidly cleared from the circulation via the kidneys and bladder, with low activity in the liver and no deposits in the intestines (Welling et al., 1999). hLF shows preferential binding to Candida albicans, a fungus. The ${ }^{99 \mathrm{~m}} \mathrm{Tc}-\mathrm{hLF}$ peptide accumulates in the gallbladder and the intestines making it unfavourable for the detection of abdominal infections (Welling et al., 2000).

\section{Timing for Imaging}

Imaging is mandatory in patients with a symptomatic prosthesis and suspicion of a "delayed" (between 3 months and 2 years after surgery) or "late" (more than 2 years after surgery) infection (according to Zimmerli et al., 2004). However, for diagnosis of an "early" (first 3 months after surgery) infection, imaging is often not necessary, since this is mostly a clinical diagnosis. The WBC scan can be performed in-patient with or without antibiotic therapy. Some authors suggested that the diagnostic accuracy of the exam increases if the patient is off-therapy for 2 weeks despite no clear data have been published supporting this theory. In figure 6, we propose a diagnostic pathway for imaging PJI. Different pathways are proposed depending on the age of prosthesis and the probability of infection.

More debated is the timing of a control scan after antibiotic therapy and the timing of a control scan after removal of an infected prosthesis with position of a temporary spacer, to verify the absence of residual infection before re-implanting a new prosthesis. These timings really depend also on the clinical organisation and local experience. Some orthopaedic surgeons re-implant a new prosthesis as soon as 2 weeks after explant of the infected prosthesis to limit the period of patient inability, without performing any control scan; more frequently, others perform a standard antibiotic therapy for 2 or 4 weeks and then wait and watch the patient for additional 2-4 weeks before re-implant, without performing any control scan; finally, others wait for a negative control scan before reimplanting a new prosthesis. In the latter case, to avoid performing unnecessary scans, it is suggested that the control scan is performed after an adequate antibiotic treatment and when clinical and laboratory parameters become negative.

Our proposed diagnostic flow chart for the proven infected prosthesis is shown in figure 7. Certainly, this is the suggested approach from the nuclear medicine point of view but no data exist in literature to support this flow chart, neither are there published studies comparing the different approaches to patient management. However, it is a matter of fact that the incidence of infection after reimplant of a prosthesis is 10 times, or more, higher than for the first implant (Cataldo et al., 2010) and no satisfactory surveillance is performed in these patients, nor are enough WBC scans requested by clinicians before re-implant of a new prosthesis.

\section{Conclusions}

The aim of this review article was to provide the clinician who deals with suspected PJI patients with an overview of the most often used nuclear medicine imaging techniques. We described their rationale of use and their limitations. Special focus was given to the most commonly used radiopharmaceuticals, i.e., ${ }^{67} \mathrm{Ga}$-citrate, ${ }^{99 \mathrm{~m}} \mathrm{Tc}$ - or ${ }^{111} \mathrm{In}$ WBC, ${ }^{99 \mathrm{~m}} \mathrm{Tc}-1 \mathrm{abelled}$ anti-granulocyte monoclonal antibodies and ${ }^{18} \mathrm{~F}$-FDG. Moreover, a brief description was given of newer promising radiopharmaceuticals, e.g. in studying the bacterial cause of the infection. Most of them are not clinically usable in clinical practice at the moment, but may be in the future.

When dealing with a patient with a symptomatic prosthesis, the clinician asks the nuclear physician to discriminate between septic or aseptic loosening of the 
device, because the treatment of the two conditions is completely different. Usually, the first approach to such patients is the three-phase bone scan (Fig. 6). This imaging technique has low costs, is largely available and highly sensible, especially when the suspicion of an infection is not too high and based on clinical signs with laboratory results (low WBC count, C-reactive protein and erythrocyte sedimentation rate). A negative bone scan excludes the possibility of any kind of loosening of the prosthesis. However, in many cases the results of the three-phase bone scan are positive which makes the diagnosis unclear. Furthermore, bone scan imaging may show positive results for 2 years after hip prosthesis placement and 5 years after knee prosthesis placement. In these cases more specific studies are required.

Scintigraphy with radiolabelled autologous WBC is considered as the first nuclear imaging method when approaching a patient with high suspicion of infection (high WBC count, CRP and ESR) during the first years after placement of the prosthesis or when the results of the three-phase bone scan are unclear. It is mandatory to obtain delayed (after 3-4 h) and late (after 20-24 h) images. No uptake at all means no infection; a weak accumulation, decreasing in time, is indicative of aseptic loosening; whereas an increase of accumulation in time indicates a septic process. Despite the high accuracy of the technique, there are some limitations: such as the laborious preparation of this radiopharmaceutical, that must be performed in sterile conditions; and the need to handle potentially contaminated blood that can result in hazard for technicians as well for patients; as well as the long time needed for the labelling procedure. To overcome these limitations, labelled antigranulocyte monoclonal antibodies may replace labelled WBC. Reports mention that antigranulocyte imaging is accurate, efficacious and safe to handle (Richter et al., 2011), but physiological uptake in the bone marrow lowers its sensitivity.

${ }^{18} \mathrm{~F}-\mathrm{FDG}$ PET is a highly accurate method for diagnosing infections, but its use is still limited because of its high costs, limited availability around the world and mainly because of its low specificity in PJI. It can be used when results of the WBC scans are equivocal, and may help to differentiate between soft tissue infection and osteomyelitis, particularly if SPECT/CT is not available for WBC. Bone marrow scintigraphy with colloids may help to differentiate between physiological uptake in the bone marrow and an infection in case of equivocal WBC scan.

In all cases, it is relevant for an accurate diagnosis to use standardised labelling procedures, image acquisition protocols and interpretation criteria. Several guidelines have been published and others are in progress from scientific societies such as EANM (Jamar et al., 2012), SNM, and IAEA.

None of the currently available radiopharmaceuticals work equally well in all situations. Ultimately, a Nuclear Medicine unit will likely have several different radiopharmaceuticals from which to choose depending on the clinical situation.

New promising approaches to the study of PJI are based on a different strategy. This strategy tries to image infection by targeting the pathogens directly involved in the infectious processes, such as bacteria, by using radiolabelled antibiotics and vitamins. However, investigations in this field are still on-going and we have to wait for the results to see if this strategy may replace WBC imaging in future or becomes an alternative for neutropenic patients.

\section{References}

Alexander K, Drost WT, Mattoon JS, Kowalski JJ, Funk JA, Crabtree AC (2005) Binding of ciprofloxacin labelled with technetium-99m versus ${ }^{99 \mathrm{~m}} \mathrm{Tc}$-pertechnetate to a live and killed equine isolate of Escherichia coil. Can J Vet Res 69: 272-277.

Akhtar MS, Imran MB, Nadeem MA, Shahid A (2012). Antimicrobial peptides as infection imaging agents: better than radiolabelled antibiotics. Int J Pept 2012: 965238.

Anguita-Alonso P, Hanssen AD, Patel R (2005) Prosthetic joint infection. Expert Rev Anti Infect Ther 3: 797-804.

Attwood PV (1995) The structure and the mechanism of action of pyruvate carboxylase. Int J Biochem Cell Biol 27: 231-249.

Baldoni D (2009) Innovative methods for the diagnosis and treatment of implant-associated infections. Doctoral Thesis, University of Basel.

Ballani N, Al-Huda FA, Khan Ha, Al-Mohannadi S, Mahmood H, Al-Enezi F (2007). The value of quantitative uptake of ${ }^{99 \mathrm{~m}}$ Tc-MDP and ${ }^{99 \mathrm{~m}}$ Tc-HMPAO white blood cells in detecting osteomyelitis in violated peripheral bones. $\mathrm{J}$ Nucl Med Technol 35: 91-95.

Becker W, Bair J, Behr T, Repp R, Streckenbach H, Beck H, Gramatzki M, Winship MJ, Goldenberg DM, Wolf F (1994) Detection of soft-tissue infections and osteomyelitis using a technetium-99m-labeled antigranulocyte monoclonal antibody fragment. J Nucl Med 35: 1436-1443.

Becker W, Meller J (2001) The role of nuclear medicine in infection and inflammation. Lancet Infect Dis 1: 326333.

Bekerman C, Hoffer PB, Bitran JD (1984) The role of gallium-67 in the clinical evaluation of cancer. Semin Nucl Med 14: 296-323.

Berbari EF, Hanssen AD, Duffy MC, Steckelberg JM, Ilstrup DM, Harmsen WS, Osmon DR (1998) Risk factors for prosthetic joint infection: case-control study. Clin Infect Dis 27: 1247-1254.

Blazeski A, Kozloff KM, Scott PJH (2010) Besilesomab for imaging inflammation and infection in peripheral bone in adults with suspected osteomyelitis. Reports Med Imaging 3: 17-27.

Britton KE, Wareham DW, Das SS, Solanki KK, Amaral H, Bhatnagar A, Katamihardja AH, Malamitsi J, Moustafa HM, Soroa VE, Sundram FX, Padhy AK (2002) Imaging bacterial infection with $(99 \mathrm{~m}) \mathrm{Tc}$-ciprofloxacin (Infecton). J Clin Pathol 55: 817-823.

Britton KE, Das SS, Solanki KK (2004) Ability of ${ }^{99 m} \mathrm{Tc}$-ciprofloxacin scintigraphy to discriminate between 
septic and sterile osteoarticular diseases. J Nucl Med 45: 922-923.

Brunenberg DE, van Steyn MJ, Sluimer JC, Bekebrede LL, Bulstra SK, Joore MA (2005) Joint recovery programme versus usual care - An economic evaluation of a clinical pathway for joint replacement surgery. Med Care 43: 1018-1026.

Cataldo MA, Petrosillo N, Cipriani M, Cauda R, Tacconelli E (2010) Prosthetic joint infection: Recent developments in diagnosis and management. J Infect $\mathbf{6 1}$ : 443-448.

Chianelli M, Boerman OC, Malviya G, Galli F, Oyen WJ, Signore A (2008) Receptor binding ligands to image infection. Curr Pharm Des 14: 3316-3325.

Datz FL (1994) Indium-111-labeled leukocytes for the detection of infection: current status. Semin Nucl Med 24: 92-109.

De Vries EF, Roca M, Jamar F, Israel O, Signore A (2010) Guidelines for the labelling of leucocytes with (99m)Tc-HMPAO. Inflammation/Infection Taskgroup of the European Association of Nuclear Medicine. Eur J Nucl Med Mol Imaging 37: 842-848.

Duff GP, Lachiewicz PF, Kelley SS (1996) Aspiration of the knee joint before revision arthroplasty. Clin Orthop Relat Res 331: 132-139.

Dumarey N, Blocklet D, Appelboom T, Tant L, Schoutens A (2002) Infecton is not specific for bacterial osteo-articular infective pathology. Eur J Nucl Med Mol Imaging 29: 530-535.

Dumarey N, Egrise D, Blocklet D, Stallenberg B, Remmelink M, del Marmol V, Van Simaeys G, Jacobs F, Goldman S (2006) Imaging infection with ${ }^{18} \mathrm{~F}-\mathrm{FDG}$-labeled leukocyte PET/CT: initial experience in 21 patients. J Nucl Med 47: 625-632.

Familiari D, Glaudemans A, Vitale V, Prosperi D, Bagni O, Lenza A, Cavallini M, Scopinaro F, Signore A (2011) Can sequential 18F-FDG PET/CT replace WBC imaging in the diabetic foot? J Nucl Med 52: 1012-1019.

Fink B, Makowiak C, Fuerst M, Berger I, Schäfer P, Frommelt L (2008) The value of synovial biopsy, joint aspiration and C-reactive protein in the diagnosis of late peri-prosthetic infection of total knee replacements. J Bone Joint Surg Br 90: 874-878.

Forstrom LA, Dunn WL, Mullan BP, Hung JC, Lowe VJ, Thorson LM (2002) Biodistribution and dosimetry of [(18)F]fluorodeoxyglucose labelled leukocytes in normal human subjects. Nucl Med Commun 23: 721-725.

Fuerst M, Fink B, Rüther W (2005) The value of preoperative knee aspiration and arthroscopic biopsy in revision total knee arthroplasty (in German). Z Orthop Ihre Grenzgeb 143: 36-41.

Genant HK, Bautovich GJ, Singh M, Lathrop KA, Harper PV (1974) Bone-seeking radionuclides: an in vivo study of factors affecting skeletal uptake. Radiology 113: 373-382.

Gemmel F, Dumarey N, Welling M (2009) Future diagnostic agents. Semin Nucl Med 39: 11-26.

Gemmel F, Van den Wyngaert H, Love C, Welling MM, Gemmel P, Palestro CJ (2012). Prosthetic joint infections: radionuclide state-of-the-art imaging. Eur J Nucl Med Mol Imaging 39: 892-909.
Goerres GW, Ziegler SI, Burger C, Berthold T, Von Schulthess GK, Buck A (2003) Artifacts at PET and PET/ $\mathrm{CT}$ caused by metallic hip prosthetic material. Radiology 226: 577-584.

Goldenberg K (1998) Septic arthritis. Lancet 351: 197 202.

Gotz F (2002) Staphylococcus and biofilms. Mol Microbiol 43: 1367-1378.

Gratz S, Reize P, Pfestroff A, Höffken H (2012) Intact versus fragmented ${ }^{99 \mathrm{~m}}$ Tc-monoclonal antibody imaging of infection in patients with septically loosened total knee arthroplasty. J Int Med Res 40: 1335-1342.

Hoffer P (1980) Gallium and infection. Jo Nucl Med 21: 484-488.

Iyengar KP, Ramesh CN, Vinjamuri S (2006) Functional imaging in orthopaedic infection-update on immunoscintigraphy. Current Orthopedics 20: 47-51.

Israel O, Keidar Z (2011) PET/CT imaging in infectious conditions. Ann N Y Acad Sci 1228: 150-166.

Jamar F, Israel O, Martin-Comin J, Buscombe J, Signore A (2012) Guideline for $\left[{ }^{18} \mathrm{~F}\right] \mathrm{FDG}$ imaging in inflammation and infection. J Nucl Med, in press.

Johnson JA, Christie MJ, Sandler MP, Parks PF Jr, Homra L, Kaye JJ (1988) Detection of occult infection following total joint arthroplasty using sequential technetium-99m HDP bone scintigraphy and indium-111 WBC imaging. J Nucl Med 29:1347-1353.

Jones-Jackson L, Walker R, Purnell G, McLaren SG, Skinner RA, Thomas JR, Suva LJ, Anaissie E, Miceli M, Nelson CL, Ferris EJ, Smeltzer MS (2005) Early detection of bone infection and differentiation from post-surgical inflammation using 2-deoxy-2-[18F]-fluoro-D-glucose positron emission tomography (FDG-PET) in an animal model. J Orthop Res 23: 1484-1489.

King AD, Peters AM, Stuttle AW, Lavender JP (1990) Imaging of bone infection with labelled white blood cells: role of contemporaneous bone marrow imaging. Eur J Nucl Med 17: 148-151.

Kumar V (2005) Radiolabeled white blood cells and direct targeting of micro-organisms for infection imaging. Q J Nucl Med Mol Imaging 49: 325-338.

Kumar V, Boddeti DK, Evans SG, Angelides S (2012) (68)Ga-citrate-PET for diagnostic imaging of infections in rats and for intra-abdominal infection in a patient. Curr Radiopharm 5: 71-75.

Larikka MJ, Ahonen AK, Junila JA, Niemela O, Hamalainen MM, Syrjala HP (2001) Extended combined ${ }^{99 \mathrm{~m}} \mathrm{Tc}$-white blood cell and bone imaging improves the diagnostic accuracy in the detection of hip replacement infections. Eur J Nucl Med 28: 288-293.

Lavender JP, Lowe J, Barker JR, Burn JI, Chaudhri MA (1971) Gallium 67 citrate scanning in neoplastic and inflammatory lesions. Br J Radiol 44: 361-366.

Lazzeri E, Erba P, Perri M, Tascini C, Doria R, Giorgetti J, Mariani G (2008) Scintigraphic imaging of vertebral osteomyelitis with ${ }^{111}$ In-biotin. Spine (Phila Pa 1976) 33: E198-E204.

Lazzeri E, Erba P, Perri M, Doria R, Tascini C, Mariani $\mathrm{G}$ (2010) Clinical impact of SPECT/CT with In-111 biotin on the management of patients with suspected spine infection. Clin Nucl Med 35: 12-17. 
Love C, Palestro CJ (2003) ${ }^{99 \mathrm{~m} T c-f a n o l e s o m a b ~ p a l a t i n ~}$ technologies. IDrugs 6: 1079-1085.

Love C, Palestro CJ (2004) Radionuclide imaging of infection. J Nucl Med Technol 32: 47-57.

Love C, Tomas MB, Marwin SE, Pugliese PV, Palestro CJ (2001) Role of nuclear medicine in diagnosis of the infected joint replacement. Radiographics 21: 1229-1238.

Love C, Marwin S, Tomas MB, Krauss ES, Tronco GG, Bhargava KK, Nichols KL, Palestro C (2004) Diagnosing infection in the failed joint replacement: a comparison of coincidence detection ${ }^{18} \mathrm{~F}-\mathrm{FDG}$ and ${ }^{111}$ In-labeled leukocyte/ ${ }^{99 \mathrm{~m}} \mathrm{Tc}$-sulfur colloid marrow imaging. J Nucl Med 45: 1864-1871.

Love C, Marwin SE, Palestro CJ (2009) Nuclear medicine and the infected joint replacement. Semin Nucl Med 39: 66-78.

Meermans G, Haddad FS (2010) Is There a role for tissue biopsy in the diagnosis of periprosthetic infection? Clin Orthop Relat Res 468: 1410-1417.

Melendez-Alafort L, Rodriguez-Cortes J, Ferro-Flores G, Arteaga de Murphy C, Herrera-Rodriguez R, Mitsoura E, Martinez-Duncker C (2004) Biokinetics of ${ }^{99 \mathrm{~m}}$ Tc-UBI 29-41 in humans. Nucl Med Biol 31: 373-379.

Nagoya S, Kaya M, Sasaki M, Tateda K, Yamashita $\mathrm{T}$ (2008) Diagnosis of peri-prosthetic infection at the hip using triple-phase bone scintigraphy. J Bone Joint Surg Br 90: $140-144$.

Nanni C, Errani C, Boriani L, Fantini L, Ambrosini V, Boschi V, Rubello D, Pettinato C, Mercuri M, Gasbarrini A, Fanti S. (2010) 68Ga-Citrate PET/CT for evaluating patients with infections of the bone: preliminary results. J Nucl Med 51: 1932-1936.

Palestro CJ, Roumanas P, Swyer AJ, Kim CK, Goldsmith SJ (1992) Diagnosis of musculoskeletal infection using combined In-111 labeled leukocyte and Tc-99m SC marrow imaging. Clin Nucl Med 17: 269-273.

Palestro CJ, Love C, Tronco GG, Tomas MB, Rini JN (2006) Combined labeled leukocyte and technetium99m sulfur colloid bone marrow imaging for diagnosing musculoskeletal infection. Radiographics 26: 859-870.

Palestro CJ, Love C, Miller TT (2007) Diagnostic imaging test and microbial infections. Cell Microbiol 9: 2323-2333.

Pelosi E, Baiocco C, Pennone M, Migliaretti G, Varetto T, Maiello A, Bellò M, Bisi G (2004) ${ }^{99 m}$ Tc-MMPAOleukocyte scintigraphy in patients with symptomatic total hip or knee arthroplasty: improved diagnostic accuracy by means of semiquantitative evaluation. J Nucl Med 45: 438-444.

Perkins PJ (1981) Early gallium-67 abdominal imaging: pitfalls due to bowel activity. AJR Am J Roentgenol 136: 1016-1017.

Peters AM (1994) The utility of [ $\left.{ }^{99 \mathrm{~m}} \mathrm{Tc}\right] \mathrm{HMPAO}-$ leukocytes for imaging infection. Semin Nucl Med 24: 110-127.

Pineda C, Espinosa R, Pena A (2009) Radiographic Imaging in osteomyelitis: the role of plain radiography, computed tomography, ultrasonography, magnetic resonance imaging, and scintigraphy. Sem Plast Surg 23: 80-89.
Prandini N, Lazzeri E, Rossi B, Erba P, Parisella MG, Signore A. (2006) Nuclear medicine imaging of bone infections. Nucl Med Commun 27: 633-644.

Reinartz P, Mumme T, Hermanns B, Cremerius U, Wirtz DC, Schaefer WM, Niethard FU, Buell U (2005). Radionuclide imaging of the painful hip arthroplasty: Positron-emission Tomography versus triple-phase bone scanning. J Bone Joint Surg Br 87-B: 465-470.

Reinartz P (2009) FDG-PET in patients with painful hip or knee arthroplasty: technical breakthrough or just more of the same. Q J Nucl Med Mol Imaging 53: 41-50.

Rennen HJ, Boerman OC, Oyen WJ, Corstens FH (2001) Imaging infection/inflammation in the new millennium. Eur J Nucl Med 28: 241-252.

Richter WS, Ivancevic V, Meller J, Lang O, Le Guludec D, Szilvazi I, Amthauer H, Chossat F, Dahmane A, Schwenke C, Signore A (2011) ${ }^{99 \mathrm{~m} T c-b e s i l e s o m a b}$ (Scintimun ${ }^{\circledR}$ ) in peripheral osteomyelitis: comparison with ${ }^{99 \mathrm{~m}}$ Tc-labelled white blood cells. Eur J Nucl Med Mol Imaging 38: 899-910.

Rini JN, Bhargava KK, Tronco GG, Singer C, Caprioli R, Marwin SE, Richardson HL, Nichols KJ, Pugliese PV, Palestro CJ (2006) PET with FDG-labeled leukocytes versus scintigraphy with ${ }^{111}$ In-oxine-labeled leukocytes for detection of infection. Radiology 238: 978-987.

Rini JN, Palestro CJ (2006) Imaging of infection and inflammation with ${ }^{18}$ F-FDG-labeled leukocytes. Q J Nucl Med Mol Imaging 50: 143-146.

Roca M, de Vries EF, Jamar F, Israel O, Signore A (2010) Guidelines for the labelling of leucocytes with (111)In-oxine. Inflammation/Infection Taskgroup of the European Association of Nuclear Medicine. Eur J Nucl Med Mol Imaging 37: 835-841.

Rusckowski M, Fritz B, Hnatowich DJ (1992) Localization of infection using streptavidin and biotin: an alternative to nonspecific polyclonal immunoglobulin. J Nucl Med 33: 1810-1815.

Rusckowski M, Gupta S, Liu G, Dou S, Hnatowich DJ (2004) Investigations of a (99m)Tc-labeled bacteriophage as a potential infection-specific imaging agent. J Nucl Med 45: 1201-1208.

Rusckowski M, Gupta S, Liu G, Dou S, Hnatowich D (2008) Investigation of four ${ }^{99 \mathrm{~m}}$ Tc-labeled bacteriophages for infection-specific imaging. Nucl Med Biol 35: 433-440.

Signore A, Glaudemans AW (2011) The molecular imaging approach to image infections and inflammation by nuclear medicine techniques. Ann Nucl Med 25: 681-700.

Signore A, Mather SJ, Piaggio G, Malviya G, Dierckx RA (2010) Molecular imaging of inflammation/infection: nuclear medicine and optical imaging agents and methods. Chem Rev 110:3112-3145.

Signore A, Glaudemans AW, Malviya G, Lazzeri E, Prandini N, Viglietti AI, De Vries EF, Dierckx RA (2012) Development and testing of a new disposable sterile device for labelling white blood cells. Q J Nucl Med Mol Imaging 56: 400-408.

Skehan SJ, White JF, Evans JW, Parry-Jones DR, Solanki CK, Ballinger JR, Chilvers ER, Peters AM (2003) Mechanism of accumulation of ${ }^{99 \mathrm{~m}} \mathrm{Tc}$-sulesomab in inflammation. J Nucl Med 44: 11-18. 
Sousa R, Massada M, Pereira A, Fontes F, Amorim I, Oliveira A (2011) Diagnostic accuracy of combined 99mTc-sulesomab and 99mTc-nanocolloid bone marrow imaging in detecting prosthetic joint infection. Nucl Med Commun 32: 834-839.

Thakur ML, McAfee JG (1984) The significance of chromosomal aberrations in indium-111-labeled lymphocytes. J Nucl Med 25: 922-927.

Tondeur MC, Sand A, Ham HH (2008) Interobserver reproducibility in the interpretation of ${ }^{99 \mathrm{~m}} \mathrm{Tc}$-labelled white blood cell scintigraphic images. Nucl Med Commun 29: 1093-1099.

Vinjamuri S, Hall AV, Solanki KK, Bomanji J, Siraj Q, O’Shaughnessy E, Das SS, Britton KE (1996) Comparison of ${ }^{99 \mathrm{~m}} \mathrm{Tc}$ infecton imaging with radiolabelled white-cell imaging in the evaluation of bacterial infection. Lancet 347: 233-235.

Von Eiff C, Jansen B, Kohnen W, Becker K (2005) Infections associatied with medical devices: pathogenesis, management and profylaxis. Drugs 65: 179-214.

Welling MM, Nibbering PH, Paulusma-Annema A, Hiemstra PS, Pauwels EK, Calame W (1999) Imaging of bacterial infections with Tc-99m-labeled human neutrophil peptide-1. J Nucl Med 40: 2073-2080.

Welling MM, Paulusma-Annema A, Balter HS, Pauwels EK, Nibbering PH (2000) Technetium-99m labelled antimicrobial peptides discriminate between bacterial infections and sterile inflammations. Eur J Nucl Med 27: 292-301.

Wilson MA (1997) Musculoskeletal system. In: Textbook of Nuclear Medicine (Wilson MA, ed). Lippincott-Raven, Philadelphia. pp 3-32.

Yao X, Wei D, Soden C, Jr., Summers MF, Beckett D (1997) Structure of the carboxy-terminal fragment of the apo-biotin carboxyl carrier subunit of Escherichia coli acetyl-CoA carboxylase. Biochemistry 36: 15089-15100.

Zhuang H, Sam JW, Chacko TK, Duarte PS, Hickeson M, Feng Q, Nakhoda KZ, Guan L, Reich P, Altimari SM, Alavi A (2003) Rapid normalization of osseous FDG uptake following traumatic or surgical fractures. Eur J Nucl Med Mol Imaging 30: 1096-1103.

Zhu X, Park MA, Gerbaudo VH, Moore SC (2007) Quantitative simultaneous In-111/Tc-99m planar imaging in a long-bone infection phantom. Phys Med Biol 52 : 7353-7365.

Zimmerli W, Waldvogel FA, Vaudaux P, Nydegger UE (1982) Pathogenesis of foreign body infection: description and characteristics of an animal model. J Infect Dis 146: 487-497.

Zimmerli W, Trampuz A, Ochsner PE (2004) Prostheticjoint infections. N Engl J Med 351: 1645-1654.

Zimmerli W (2006) Prosthetic-joint-associated infections. Best Pract Res Clin Rheum 20: 1045-1063.

\section{Discussion with Reviewers}

Reviewer I: Is there any need for radionuclide imaging techniques for imaging the suspected prosthetic joint infection? If yes, as a 'screening tool' or either as a more 'tailored tool' in combination with ESR/CRP levels?

Authors: This is described in the flow chart of Fig. 6. There is need for radionuclide imaging techniques when there is a clinical suspicion and when X-ray and laboratory parameters are also suspect. However, as the reviewer may well know, there are cases in which despite negativity of blood test the scan can be positive, particularly in chronic osteomyelitis and/or after antibiotic therapy. In these cases the scan is useful.

Reviewer I: Is there any incremental value of hybrid SPECT/CT and PET/CT technique for PJI imaging?

Authors: Yes, there is. We all know that some artefacts in CT may arise due to the metal prosthesis; however, it is possible to combine physiology and anatomy with these hybrid techniques, thereby making differentiation between bone infections, infections in the space between bone and soft tissue, and soft tissue infections.

Reviewer I: Is there a need for a non-invasive radionuclide technique for therapy monitoring in PJI; which technique and when to use it?

Authors: As stated in the section on Timing for Imaging and in the flow chart of Fig. 7, there is absolutely a need for an imaging technique that helps the clinician to decide when to stop antibiotics and what is the best timing for surgery and replacement. We thereby propose the flow chart in Fig. 7 to be useful for this timing management; however, we know this is not supported by literature at the moment, and maybe other/better imaging techniques are available.

Reviewer II: Regarding Fig. 7, in clinical practice in revision surgeries an antibiotic therapy/ debridement/ placement of a temporal spacer etc. shall affect WBS scans toward false-positives. Could you comment on this?

Authors: The reviewer is absolutely right about how antibiotic therapy affects WBS scans; however, this may cause false-negative scans. Therefore, we advise 2 weeks of wait and watch (so, no antibiotic therapy) before performing WBC scintigraphy. Debridement and placement of a temporal spacer may cause false-positive scans. However, a total of 6 weeks (4 weeks antibiotics and 2 weeks wait and watch) or more between the surgery and placement of a spacer and WBC scintigraphy should be enough to avoid this.

Reviewer II: Regarding your statement in the Conclusions, I thought that FDG-PET is a more sophisticated and expensive technique than SPECT, and a radiology centre would rather have a SPECT than FDG/PET facility.

Authors: The reviewer is right, FDG-PET is more sophisticated and expensive than SPECT-CT. However, for diagnosing PJI, WBC scintigraphy with SPECT-CT is more sensitive and specific than FDG-PET that could be positive due to many causes. Therefore, first choice would be WBC scintigraphy. In our opinion, radiology centres are more often equipped with PET/CT than with SPECT/CT. Nuclear medicine centres usually have both cameras available. 